\title{
SCOPELLARIA, A NEW GENUS NAME IN CUCURBITACEAE
}

\author{
W.J.J.O. DE WILDE \& B.E.E. DUYFJES \\ Nationaal Herbarium Nederland, Universiteit Leiden branch, P.O. Box 9514, \\ 2300 RA Leiden, The Netherlands; e-mail: dewilde@nhn.leidenuniv.nl
}

With the publication of the redefinition of the genus Zehneria and some resembling genera in SE Asia, it appeared that the name Scopella W.J. de Wilde \& Duyfjes is a homonym of the older Scopella Mains (1939). Therefore, a new name for the genus Scopella and the necessary new combinations for its specific and intraspecific taxa are proposed. For a key to these and their descriptions see De Wilde \& Duyfjes (2006).

Scopellaria W.J. de Wilde \& Duyfjes, nom. nov. - Scopella W.J. de Wilde \& Duyfjes (2006) 34, non Mains (1939), homonym. - Type: Scopellaria marginata (Blume) W.J. de Wilde \& Duyfjes.

Scopellaria diversifolia (Merr.) W.J. de Wilde \& Duyfjes, comb. nov. - Melothria diversifolia Merr. (1923) 44. - Scopella diversifolia (Merr.) W.J. de Wilde \& Duyfjes (2006) 34. - Lectotype (De Wilde \& Duyfjes 2006: 34): Ramos 1896 (BO, UC).

Scopellaria marginata (Blume) W.J. de Wilde \& Duyfjes, comb. nov. - Bryonia marginata Blume (1826) 924. - Scopella marginata (Blume) W.J. de Wilde \& Duyfjes (2006) 35. - Type: Blume 920 (L).

a. var. marginata

Informal form 'marginata'

Informal form 'affinis'

b. var. penangense (C.B. Clarke) W.J. de Wilde \& Duyfjes, comb. nov. - ?Cerasiocarpum penangense C.B. Clarke (1879) 629. - Scopella marginata (Blume) W.J. de Wilde \& Duyfjes var. penangense (C.B. Clarke) W.J. de Wilde \& Duyfjes (2006) 38. - Type: Wallich 6704 (K-W).

\section{ACKNOWLEDGEMENTS}

We are grateful to Mrs. Gea Zijlstra (U) for informing us about the illegitimacy of Scopella in Cucurbitaceae and to Jan-Frits Veldkamp (L) for advice. 


\section{REFERENCES}

Blume, C.L. 1826. Bijdragen tot de Flora van Nederlandsch Indië 15: 922-940. Ter Lands Drukkerij, Batavia.

Clarke, C.B. 1879. Cucurbitaceae. In: J.D. Hooker, The Flora of British India 2: 604-635. Reeves \& Co., London.

De Wilde, W.J.J.O. \& B.E.E. Duyfjes. 2006. Redefinition of Zehneria and four new related genera (Cucurbitaceae) with an enumeration of the Australasian and Pacific species. Blumea 51: 1-88. Mains, E.B. 1939. Scopella gen. nov. of the Pucciniaceae. Ann. Mycol. 37: 58.

Merrill, E.D. 1923. New or noteworthy Bornean plants. J. Malayan Branch Roy. Asiat. Soc. 1: 44-45. 